\title{
Factors Affecting Graft Survival among Patients Receiving Kidneys from Live Donors: A Single-Center Experience
}

\author{
Mohamed A. Ghoneim, ${ }^{1}$ Mohamed A. Bakr, ${ }^{2}$ Ayman F. Refaie, ${ }^{2}$ Ahmed I. Akl, ${ }^{2}$ \\ Ahmed A. Shokeir, ${ }^{1}$ Ahmed B. Shehab El-Dein, ${ }^{1}$ Hesham M. Ammar, ${ }^{2}$ Amani M. Ismail, ${ }^{3}$ \\ Hussein A. Sheashaa, ${ }^{2}$ and Mahmoud A. El-Baz ${ }^{4}$ \\ ${ }^{1}$ Department of Urology, The Urology \& Nephrology Center, Mansoura, Egypt \\ ${ }^{2}$ Division of Nephrology, The Urology \& Nephrology Center, Mansoura, Egypt \\ ${ }^{3}$ Division of Immunology, The Urology \& Nephrology Center, Mansoura, Egypt \\ ${ }^{4}$ Division of Pathology, The Urology \& Nephrology Center, Mansoura, Egypt
}

Correspondence should be addressed to Mohamed A. Ghoneim; ghoneimma@yahoo.com

Received 27 April 2013; Revised 11 June 2013; Accepted 11 June 2013

Academic Editor: Gerald Brandacher

Copyright (C) 2013 Mohamed A. Ghoneim et al. This is an open access article distributed under the Creative Commons Attribution License, which permits unrestricted use, distribution, and reproduction in any medium, provided the original work is properly cited.

Introduction. The aim of this report is to study the graft and patient survival in a large cohort of recipients with an analysis of factors that may affect the final outcomes. Methods. Between March 1976 and March 2008, 1967 consecutive live-donor renal transplants were carried out. Various variables that may have an impact on patients and/or graft survival were studied in two steps. Initially, a univariate analysis was carried out. Thereafter, significant variables were embedded in a stepwise regression analysis. Results. The overall graft survival was $86.7 \%$ and $65.5 \%$, at 5 and 10 years, respectively. The projected half-life for grafts was 17.5 years and for patients was 22 years. Five factors had an independent negative impact on graft survival: donor's age, genetic considerations, the type of primary immunosuppression, number of acute rejection episodes, and total steroid dose during the first 3 months after transplantation. Conclusions. Despite refinements in tissue matching techniques and improvements in immunosuppression protocols, an important proportion of grafts is still lost following living donor kidney transplantation, presumably due to chronic allograft nephropathy.

\section{Introduction}

In March 1976, the first renal transplantation in Egypt was carried out at the Department of Urology, University of Mansoura. A mother donated one of her kidneys to her daughter who was suffering from end-stage renal disease secondary to chronic pyelonephritis. Armed only with azathioprine and corticosteroids, the operative procedure and the functional outcome were very successful. A typical example of beginner's luck. Following a very slow start, the number of procedures increased gradually until it has currently reached a rate exceeding 80 cases every year. Herein, our long-term results are reported with an analysis of factors which can have an impact on patients and graft survival.

\section{Patients and Methods}

2.1. Patients. Between March 1976 and March 2008, 1967 consecutive living donor renal transplants were carried out at the Urology \& Nephrology Center, Mansoura University, Egypt. There were 1620 related and 347 unrelated donors. For recipients, our exclusion criteria included sensitization with positive lymphocytotoxic cross match and donor specific antibodies, recent malignancy, addiction, psychiatric disorders, type I diabetes mellitus, and significant extrarenal organs failure (pulmonary, hepatic, and cardiac). Absolute contraindications for donation included active infections, diabetes, even minimal renal function impairment, arterial hypertension, and serological positivity for HBV or 
HCV. Over the years, different immunosuppression regimens were utilized with or without an induction treatment. Azathioprine-based therapy was initially employed. Later on, a cyclosporine-based protocol was adopted [1]. Subsequently, triple immunosuppression regimens were utilized [2] Currently, steroid and/or calcineurin inhibitor-free protocols are the subject of several clinical trials $[3,4]$.

Approval of our Institutional Review Board (IRB) Committee was obtained.

2.2. Follow-Up. Patients were closely and regularly followed up for evaluation of renal function, the onset of surgical or medical complications, and side effects of immunosuppression. The median observation period was 7.47 years (range: $0-31.15$ years).

2.3. Statistical Analysis. Actuarial survival was estimated by the Kaplan-Meier method. The log derivation of the percent survival was utilized for construction of graphs to predict the half-life of grafts and/or patients. Univariate analysis was carried out to correlate the graft survival to various preoperative, operative, and postoperative variables. Differences in survival were determined by the log-rank test. A $P$ value of $\leq 0.05$ was considered significant. For the determination of those factors who had an independent impact on graft survival, a Cox proportional hazard analysis was utilized. The variables included in this analysis were only those which were significant with the univariate study.

\section{Results}

The overall actuarial graft survival was $86.7 \%$ and $65.5 \%$ at 5 and 10 years, respectively. The corresponding patient survival was $89.7 \%$ and $77.8 \%$ (Figure 1). The graft survival was essentially stable throughout the first 5 years. A negative and steady decline was observed thereafter. The projected half-life for grafts was 17.5 years and for recipients was 22 years (Figure 2).

Of the demographic variables, 3 had a significant negative impact on graft survival: donor's sex, donor's age, and recipient's age (Table $1(\mathrm{a})$ ). Our data suggest that recipients who did not receive a blood transfusion had a better outcome (Table 1(b)). The number of class I and/or class II mismatches had a significant negative impact on graft survival. When both classes were pooled together, 2 observations could be made: the greater the number of mismatches the poorer was the result. Furthermore, there was a very clear separation in the prognostic outcome if the total number of mismatches was 2 or less versus 3 or more. It was also noted that HLAidentical siblings had the best short- and long-term graft and patient survival (Table $1(\mathrm{c})$ ). The nature of the original kidney disease, if it was identified, had a marginal negative impact on graft survival. Other current or past medical disorders did not have an influence (Table $1(\mathrm{~d})$ ).

Of the technical variables, 2 had a significant effect on graft survival: the time to diuresis and the total ischemia time (Table 2). Four post-transplant factors had a significant impact on graft survival: the primary immunosuppression

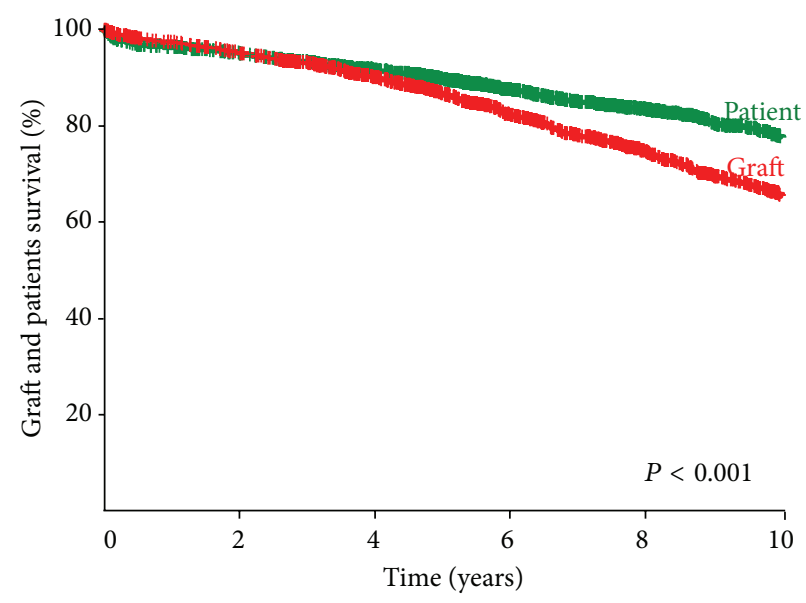

FIgURE 1: Actuarial patient and graft survival. Patient survival was $89.7 \pm 0.7 \%$ and $77.8 \pm 1.2 \%$ years at 5 and 10 years, respectively. Graft survival was $86.7 \pm 0.8 \%$ at 5 years and dropped to $65.5 \pm 1.3 \%$ at 10 years.

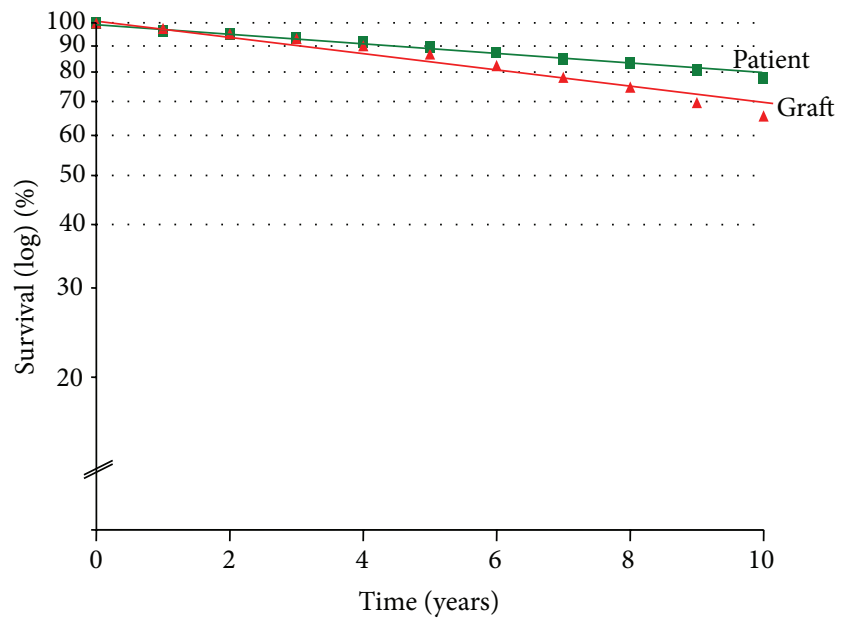

FIGURE 2: Log derivation of percentage of survival. The projected half-life for patients was 22 years and for grafts was 17.5 years.

(Figure 3), the number of acute rejection episodes, the total dose of steroid during the first 3 months, and post-transplant hypertension (Table 3 ).

Of all the factors which had a significant impact on graft survival by univariate analysis, only 5 sustained their significance when the step-wise regression analysis was carried out (Table 4). In this analysis, evidence was provided that donor's age, genetic considerations, primary immunosuppression, number of acute rejection episodes, and total steroid dose during the first 3 months acted as independent variables which had a significant influence on graft survival.

\section{Discussion}

Over recent years, there has been a global increase in the number of live-donor kidney transplants in view of a severe shortage in the availability of deceased donor 
TABLE 1: (a) Graft survival relative to pretransplantation variables: personal factors. (b) Graft survival relative to pretransplantation variables: hematological factors. (c) Graft survival relative to pre-transplantation variables: immunologic factors. (d) Graft survival relative to pretransplantation variables: current and past medical disorders.

(a)

\begin{tabular}{|c|c|c|c|c|c|c|c|c|}
\hline & $\begin{array}{c}\text { No. of } \\
\text { patients }\end{array}$ & $\begin{array}{c}5 \text {-year } \\
\text { survival \% }\end{array}$ & \multicolumn{2}{|c|}{$95 \%$ CI } & $\begin{array}{c}10 \text {-year } \\
\text { survival \% }\end{array}$ & \multicolumn{2}{|c|}{$95 \%$ CI } & $\begin{array}{l}P \text {-value } \\
\text { (log rank) }\end{array}$ \\
\hline Male & 944 & 88.6 & 86.44 & 90.76 & 69.9 & 66.37 & 73.43 & \multirow{2}{*}{0.012} \\
\hline Female & 1023 & 85.0 & 82.65 & 87.35 & 61.5 & 57.97 & 65.03 & \\
\hline \multicolumn{9}{|l|}{ Donor's age (years) } \\
\hline$\leq 30$ & 772 & 87.7 & 85.35 & 90.05 & 68.5 & 64.58 & 72.42 & \multirow{4}{*}{$<0.001$} \\
\hline $31-40$ & 627 & 87.9 & 85.16 & 90.64 & 69.6 & 65.29 & 73.91 & \\
\hline $41-50$ & 389 & 83.4 & 79.48 & 87.32 & 58.0 & 52.12 & 63.88 & \\
\hline$\geq 50$ & 179 & 85.3 & 79.81 & 90.79 & 52.9 & 43.30 & 62.50 & \\
\hline \multicolumn{9}{|l|}{ Recipient's sex } \\
\hline Male & 1466 & 87.6 & 85.84 & 89.46 & 64.7 & 54.9 & 74.50 & \multirow{2}{*}{0.829} \\
\hline Female & 501 & 84.3 & 80.97 & 87.63 & 67.8 & 63.10 & 72.50 & \\
\hline \multicolumn{9}{|c|}{ Recipient's age (years) } \\
\hline$\leq 20$ & 378 & 84.4 & 80.68 & 88.12 & 59.0 & 52.92 & 65.08 & \multirow{5}{*}{0.002} \\
\hline $21-30$ & 709 & 88.6 & 86.25 & 90.95 & 66.9 & 62.78 & 71.02 & \\
\hline $31-40$ & 556 & 86.5 & 83.56 & 89.44 & 67.0 & 62.49 & 71.51 & \\
\hline $41-50$ & 270 & 85.7 & 81.39 & 90.01 & 67.4 & 60.54 & 74.26 & \\
\hline$\geq 50$ & 54 & 86.7 & 75.53 & 97.87 & 71.2 & 52.78 & 89.62 & \\
\hline \multicolumn{9}{|c|}{ The sex donor-recipient } \\
\hline Male-male & 693 & 89.5 & 87.15 & 91.85 & 68.2 & 63.89 & 72.51 & \multirow{4}{*}{0.082} \\
\hline Male-female & 251 & 86.1 & 81.59 & 90.61 & 74.4 & 68.13 & 80.67 & \\
\hline Female-male & 773 & 85.9 & 83.35 & 88.45 & 61.5 & 57.38 & 65.62 & \\
\hline Female-female & 250 & 82.6 & 77.70 & 87.50 & 61.3 & 54.24 & 68.36 & \\
\hline \multicolumn{9}{|l|}{ Donor relationship } \\
\hline Related & 1620 & 87.3 & 85.54 & 89.06 & 65.7 & 62.96 & 68.44 & \multirow{2}{*}{0.651} \\
\hline Unrelated & 347 & 84.3 & 80.18 & 88.42 & 64.6 & 58.33 & 70.87 & \\
\hline
\end{tabular}

(b)

\begin{tabular}{|c|c|c|c|c|c|c|c|c|}
\hline & $\begin{array}{c}\text { No. of } \\
\text { patients }\end{array}$ & $\begin{array}{c}5 \text {-year } \\
\text { survival \% }\end{array}$ & \multicolumn{2}{|c|}{$95 \%$ CI } & $\begin{array}{c}10 \text {-year } \\
\text { survival \% }\end{array}$ & \multicolumn{2}{|c|}{$95 \% \mathrm{CI}$} & $\begin{array}{l}P \text { value } \\
\text { (log rank) }\end{array}$ \\
\hline & & \multicolumn{7}{|c|}{ ABO compatibility } \\
\hline Identical & 1579 & 87.3 & 85.54 & 89.06 & 65.8 & 63.06 & 68.54 & \multirow{2}{*}{0.500} \\
\hline Different compatible & 388 & 84.5 & 80.78 & 88.22 & 64.4 & 58.72 & 70.08 & \\
\hline \multicolumn{9}{|l|}{ Type of blood transfusion } \\
\hline No transfusion & 1049 & 89.1 & 87.14 & 89.80 & 69.0 & 65.28 & 72.72 & \multirow{3}{*}{$<0.001$} \\
\hline Third party & 906 & 84.2 & 81.65 & 83.03 & 62.8 & 59.47 & 66.13 & \\
\hline Donor specific & 12 & - & & & - & & & \\
\hline \multicolumn{9}{|l|}{ No. of blood transfusion } \\
\hline No transfusion & 1049 & 89.1 & 87.14 & 89.80 & 68.8 & 65.08 & 72.52 & \multirow{4}{*}{0.001} \\
\hline 1-2 times & 301 & 87.1 & 83.18 & 91.06 & 67.3 & 61.42 & 73.18 & \\
\hline $3-4$ & 265 & 82.1 & 77.40 & 86.80 & 64.4 & 58.13 & 70.67 & \\
\hline$\geq 5$ & 352 & 83.3 & 79.18 & 87.42 & 57.8 & 52.31 & 63.29 & \\
\hline
\end{tabular}

(c)

\begin{tabular}{|c|c|c|c|c|c|c|c|c|}
\hline HLA-A, B (class I) & $\begin{array}{c}\text { No. of } \\
\text { patients }\end{array}$ & $\begin{array}{c}5 \text {-year } \\
\text { survival \% }\end{array}$ & \multicolumn{2}{|c|}{$95 \% \mathrm{CI}$} & $\begin{array}{c}10 \text {-year } \\
\text { survival \% }\end{array}$ & \multicolumn{2}{|c|}{$95 \% \mathrm{CI}$} & $\begin{array}{l}P \text { value } \\
\text { (log rank) }\end{array}$ \\
\hline 0-MM & 153 & 93.2 & 89.08 & 97.32 & 71.4 & 62.38 & 80.42 & \multirow{5}{*}{$<0.001$} \\
\hline $1-\mathrm{MM}$ & 229 & 92.6 & 89.07 & 96.13 & 74.1 & 67.44 & 80.76 & \\
\hline 2-MM & 996 & 86.2 & 84.04 & 88.36 & 62.7 & 59.17 & 66.23 & \\
\hline 3-MM & 309 & 82.5 & 77.99 & 87.01 & 62.4 & 56.13 & 68.67 & \\
\hline 4-MM & 132 & 85.7 & 79.43 & 91.97 & 71.0 & 62.18 & 79.82 & \\
\hline
\end{tabular}


(c) Continued.

\begin{tabular}{|c|c|c|c|c|c|c|c|c|}
\hline & $\begin{array}{c}\text { No. of } \\
\text { patients }\end{array}$ & $\begin{array}{c}\text { 5-year } \\
\text { survival \% }\end{array}$ & \multicolumn{2}{|c|}{$95 \% \mathrm{CI}$} & $\begin{array}{c}10 \text {-year } \\
\text { survival \% }\end{array}$ & \multicolumn{2}{|c|}{$95 \% \mathrm{CI}$} & $\begin{array}{l}P \text { value } \\
\text { (log rank) }\end{array}$ \\
\hline 0-MM & 201 & 91.5 & 87.58 & 95.42 & 77.6 & 69.56 & 85.64 & \multirow{3}{*}{0.010} \\
\hline $1-\mathrm{MM}$ & 1720 & 86.7 & 84.94 & 88.46 & 64.5 & 61.76 & 67.24 & \\
\hline 2-MM & 2 & - & & & - & & & \\
\hline \multicolumn{9}{|l|}{ HLA (class I + II) MM } \\
\hline $0-\mathrm{MM}$ & 88 & 94.1 & 89.0 & 99.20 & 79.6 & 67.84 & 91.36 & \multirow{6}{*}{0.001} \\
\hline $1-\mathrm{MM}$ & 102 & 93.8 & 89.09 & 98.50 & 68.0 & 57.02 & 78.98 & \\
\hline 2-MM & 234 & 90.0 & 86.08 & 93.92 & 71.3 & 64.64 & 77.96 & \\
\hline 3-MM & 968 & 86.2 & 83.85 & 88.55 & 62.8 & 59.08 & 66.52 & \\
\hline 4-MM & 290 & 82.4 & 77.89 & 86.91 & 62.0 & 55.53 & 68.47 & \\
\hline $5-\mathrm{MM}$ & 131 & 85.6 & 79.33 & 91.87 & 69.3 & 60.28 & 78.32 & \\
\hline \multicolumn{9}{|l|}{$\begin{array}{l}\text { HLA-A, B, DR (class I and II) } \\
\text { MM }\end{array}$} \\
\hline MM & & & & & & & & \\
\hline $0 / 1 / 2$ & 424 & 91.8 & 89.06 & 94.54 & 72.1 & 67.00 & 77.20 & \multirow{2}{*}{0.011} \\
\hline $3 / 4 / 5 / 6$ & 1389 & 85.8 & 83.44 & 87.36 & 63.4 & 60.46 & 66.34 & \\
\hline \multicolumn{9}{|l|}{ Genetic considerations } \\
\hline HLA-ID sibling & 152 & 94.4 & 92.64 & 96.16 & 79.2 & 70.18 & 88.22 & \multirow{3}{*}{0.005} \\
\hline 1-Haplotype MM (R) & 1322 & 87.7 & 85.94 & 89.46 & 64.7 & 61.56 & 67.84 & \\
\hline 2-Haplotype MM (R + UR) & 449 & 83.2 & 79.48 & 86.92 & 64.0 & 58.51 & 69.49 & \\
\hline
\end{tabular}

(d)

\begin{tabular}{|c|c|c|c|c|c|c|c|c|}
\hline Bilharziasis & $\begin{array}{c}\text { No. of } \\
\text { patients }\end{array}$ & $\begin{array}{c}5 \text {-year } \\
\text { survival \% }\end{array}$ & \multicolumn{2}{|c|}{$95 \% \mathrm{CI}$} & $\begin{array}{c}10 \text {-year } \\
\text { survival \% }\end{array}$ & \multicolumn{2}{|c|}{$95 \%$ CI } & $\begin{array}{l}P \text { value } \\
\text { (log rank) }\end{array}$ \\
\hline (i) Negative & 1442 & 87.6 & 85.84 & 89.36 & 65.9 & 62.76 & 69.04 & \multirow{2}{*}{0.796} \\
\hline (ii) Positive & 525 & 84.6 & 81.46 & 87.74 & 64.4 & 59.89 & 68.91 & \\
\hline \multicolumn{9}{|l|}{ Original kidney diseases } \\
\hline (i) Glomerulonephritis & 214 & 82.8 & 73.74 & 88.09 & 63.5 & 56.05 & 70.95 & \multirow{9}{*}{0.020} \\
\hline (ii) Chronic pyelonephritis & 259 & 90.6 & 86.88 & 94.32 & 73.3 & 67.22 & 79.38 & \\
\hline (iii) Nephrosclerosis & 50 & 84.9 & 74.51 & 95.29 & 55.4 & 39.72 & 71.08 & \\
\hline (iv) Obstructive uropathy & 57 & 92.2 & 84.95 & 99.45 & 76.0 & 61.50 & 90.50 & \\
\hline (v) Amyloidosis & 33 & 93.9 & 85.67 & 102.13 & 80.4 & 64.52 & 96.28 & \\
\hline (vi) Congenital polycystic & 51 & 93.4 & 86.15 & 100.65 & 55.8 & 37.57 & 74.03 & \\
\hline (vii) Hypoplasia & 17 & 100.0 & 100.00 & 100.00 & 83.1 & 61.54 & 104.66 & \\
\hline (viii) Others & 126 & 80.9 & 73.65 & 88.15 & 58.8 & 45.67 & 71.93 & \\
\hline (ix) Not determined & 1160 & - & - & - & - & - & - & \\
\hline \multicolumn{9}{|l|}{ Types of glomerulonephritis (GN) } \\
\hline (i) Mesangiocapillary GN & 35 & 81.8 & 68.67 & 94.93 & 62.7 & 42.90 & 82.50 & \multirow{6}{*}{0.701} \\
\hline (ii) Membranous GN & 22 & 57.8 & 36.83 & 78.77 & 57.8 & 36.83 & 78.77 & \\
\hline (iii) F.S.G.S. & 70 & 90.7 & 83.64 & 97.76 & 66.6 & 53.66 & 79.54 & \\
\hline (iv) Mesangioproliferative GN & 23 & 73.9 & 55.87 & 91.93 & 67.8 & 53.66 & 79.54 & \\
\hline (v) Crescentic GN & 16 & 87.5 & 71.23 & 103.77 & 46.7 & 17.69 & 75.71 & \\
\hline (vi) Hereditary nephritis & 48 & 87.0 & 77.20 & 96.80 & 65.7 & 49.80 & 79.60 & \\
\hline \multicolumn{9}{|l|}{ Pretransplant hypertension } \\
\hline (i) Normotensive & 826 & 87.2 & 84.85 & 89.55 & 64.6 & 60.48 & 68.72 & \multirow{2}{*}{0.466} \\
\hline (ii) Hypertensive & 1141 & 86.4 & 84.24 & 88.56 & 66.1 & 62.77 & 69.43 & \\
\hline \multicolumn{9}{|l|}{ No. of transplants received } \\
\hline (i) First & 1891 & 86.0 & 85.03 & 88.17 & 65.1 & 62.55 & 67.65 & \multirow{3}{*}{0.242} \\
\hline (ii) Second & 73 & 90.0 & 82.94 & 97.06 & 77.8 & 67.22 & 88.38 & \\
\hline (iii) Third & 3 & - & - & - & - & & & \\
\hline
\end{tabular}


TABLE 2: Graft survival relative to technical variables.

\begin{tabular}{|c|c|c|c|c|c|c|c|c|}
\hline No. of renal arteries & $\begin{array}{c}\text { No. of } \\
\text { patients }\end{array}$ & $\begin{array}{c}5 \text {-year } \\
\text { survival \% }\end{array}$ & \multicolumn{2}{|c|}{$95 \% \mathrm{CI}$} & $\begin{array}{c}10 \text {-year } \\
\text { survival \% }\end{array}$ & \multicolumn{2}{|c|}{$95 \% \mathrm{CI}$} & $\begin{array}{l}P \text { value } \\
\text { (log rank) }\end{array}$ \\
\hline (i) Single & 1749 & 86.8 & 85.23 & 88.37 & 66.0 & 63.26 & 68.74 & \multirow{2}{*}{0.474} \\
\hline (ii) Multiple & 218 & 86.1 & 81.20 & 91.00 & 61.0 & 52.38 & 69.62 & \\
\hline \multicolumn{9}{|l|}{ Total ischemia time (min) } \\
\hline$<30$ & 225 & 89.3 & 85.18 & 93.42 & 61.4 & 54.74 & 68.06 & \multirow{3}{*}{0.039} \\
\hline $30-60$ & 1506 & 86.2 & 84.44 & 87.96 & 65.7 & 62.76 & 68.64 & \\
\hline$>60$ & 236 & 87.8 & 83.29 & 92.31 & 74.2 & 66.56 & 81.84 & \\
\hline \multicolumn{9}{|l|}{ Time to diuresis } \\
\hline (i) Immediate $(<10 \mathrm{~min})$ & 1806 & 87.2 & 85.63 & 88.77 & 66.0 & 63.45 & 68.55 & \multirow{2}{*}{0.029} \\
\hline (ii) Delayed (>10 min) & 161 & 81.5 & 75.03 & 87.97 & 59.2 & 49.99 & 68.41 & \\
\hline \multicolumn{9}{|l|}{ Primary urinary recontinuity } \\
\hline (i) Ureterovesical (Lead better) & 170 & 77.1 & 70.44 & 83.76 & 57.8 & 49.76 & 65.84 & \\
\hline (ii) Ureterovesical (Leich Grieg) & 1761 & 87.5 & 85.93 & 89.07 & 66.0 & 63.26 & 68.74 & \\
\hline (iii) Ureteroureteral & 29 & 92.0 & 81.42 & 102.58 & 74.7 & 57.06 & 92.34 & 0.624 \\
\hline (iv) Pyeloureteral & 3 & - & - & - & - & \multicolumn{2}{|c|}{-} & \\
\hline (v) Ileal conduit & 4 & - & - & - & - & \multicolumn{2}{|c|}{-} & \\
\hline
\end{tabular}

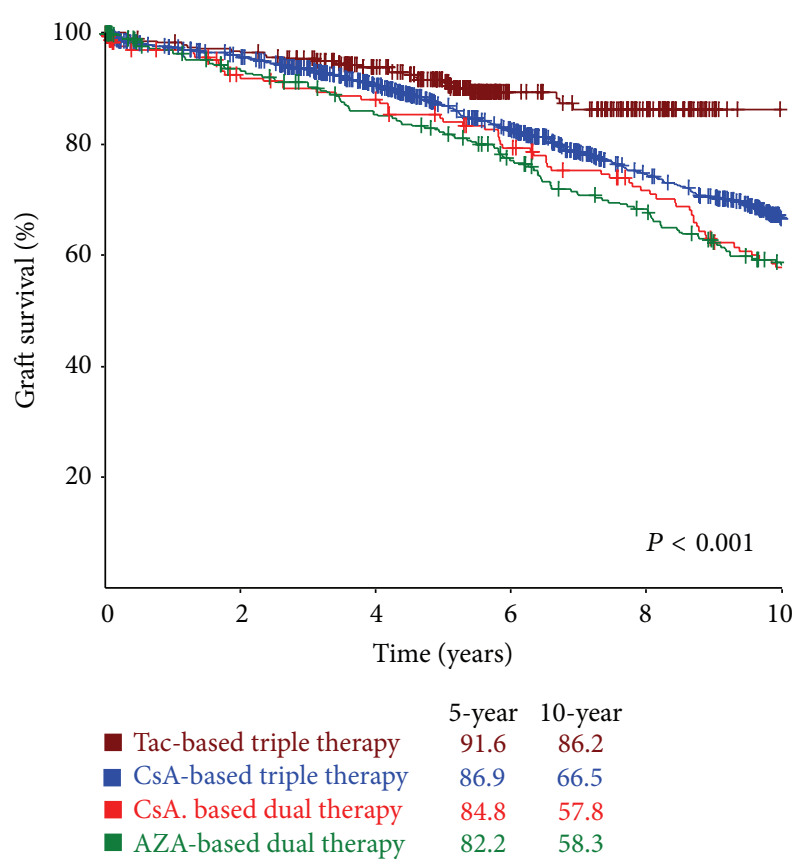

FIGURE 3: Graft survival relative to primary immunosuppressive regimen. Univariate analysis indicated that Tac-based triple therapy provided the best outcome.

organs $[5,6]$. The results of live-donor transplantation are generally superior to those obtained from deceased donors [7]. This can be explained, at least partially, by the short ischemia time. Factors inherent to the transplanted organ itself can also play an important role. Live donors are subject to rigorous predonation assessment. Furthermore, they are not exposed to major cardiovascular instability, sepsis, or nephrotoxic agents that may occur during hospitalization before declaration of brain death [8]. Our study relies only on an analysis of results of living donation. The overall graft survival was $86.7 \%$ and $65.5 \%$ at 5 and 10 years, respectively, with a projected half-life of 17.5 years. This compares favorably with recently published data [7, 9]. Out of the 1967 donors, 347 were unrelated $(16.7 \%)$. The probability of graft survival among related and unrelated donors was essentially similar. This is consistent with previously published reports [10]. It is of interest to note that the use of unrelated donors led to a higher graft survival than related ones in certain conditions, namely, type I DM, focal glomerulosclerosis and polycystic kidney disease [11].

In our report, factors that can possibly have an influence on graft and patient survival were evaluated by univariate as well as by multivariate analysis. Out of all the studied variables, only 5 had an independent impact on the graft survival: the donor's age, the genetic considerations, the type of primary immunosuppression, the number of acute rejection episodes, and the total dose of steroids during the first 3 months after transplantation. It is noteworthy that the postoperative urologic complications in this series did not have a significant impact on patient and graft survival. The nature of such complications and their impact on patient and graft survival had been fully addressed in a previous report [12].

There is some controversy regarding the impact of the donor's age on the outcome of renal transplantation from living donors. In our series, there was a negative impact on graft survival of kidneys obtained from donors above the age of 40 years. Similar observations were also reported by several investigators $[9,13,14]$. Reduced pre-donation GFR, higher blood pressure, and total cholesterol levels, usually associated with older age, were suggested as a possible 
TABLE 3: Graft survival relative to posttransplantation variables.

\begin{tabular}{|c|c|c|c|c|c|c|c|c|}
\hline Induction therapy & $\begin{array}{c}\text { No. of } \\
\text { patients }\end{array}$ & $\begin{array}{c}5 \text {-year } \\
\text { survival \% }\end{array}$ & \multicolumn{2}{|c|}{$95 \% \mathrm{CI}$} & $\begin{array}{c}10 \text {-year } \\
\text { survival \% }\end{array}$ & \multicolumn{2}{|c|}{$95 \% \mathrm{CI}$} & $\begin{array}{l}P \text { value } \\
(\log \text { rank) }\end{array}$ \\
\hline No & 1108 & 86.5 & 84.34 & 88.66 & 64.6 & 61.66 & 67.54 & \multirow{2}{*}{0.536} \\
\hline Yes & 859 & 87.1 & 84.75 & 89.45 & 69.5 & 64.80 & 74.20 & \\
\hline \multicolumn{9}{|l|}{ Primary immunosuppression } \\
\hline (i) Aza-based (dual therapy) & 309 & 82.2 & 77.69 & 86.71 & 58.3 & 52.42 & 64.18 & \multirow{5}{*}{$<0.001$} \\
\hline (ii) CsA-based (dual therapy) & 303 & 84.8 & 79.12 & 90.48 & 57.8 & 49.76 & 65.84 & \\
\hline (iii) CsA-based (triple therapy) & 988 & 86.9 & 84.74 & 89.06 & 66.5 & 63.36 & 69.64 & \\
\hline (iv) Tacrolimus-based (triple therapy) & 217 & 91.6 & 88.27 & 94.93 & 86.2 & 80.91 & 91.49 & \\
\hline (v) Sirolimus-based therapy & 85 & 88.9 & 82.04 & 95.76 & - & & & \\
\hline \multicolumn{9}{|l|}{$\begin{array}{l}\text { Total steroid dose } \\
\text { (during first } 3 \text { months) }\end{array}$} \\
\hline$<5 \mathrm{gm}$ & 1158 & 89.3 & 87.34 & 91.26 & 70.3 & 66.77 & 73.83 & \multirow{3}{*}{$<0.001$} \\
\hline $5-10 \mathrm{gm}$ & 601 & 85.0 & 82.06 & 87.94 & 64.8 & 60.68 & 68.92 & \\
\hline$>10 \mathrm{gm}$ & 208 & 77.9 & 72.02 & 83.78 & 46.9 & 39.65 & 54.15 & \\
\hline \multicolumn{9}{|l|}{$\begin{array}{l}\text { No. of acute rejection episodes } \\
\text { (during first } 3 \text { months) }\end{array}$} \\
\hline (i) No & 708 & 94.2 & 92.44 & 95.96 & 82.1 & 78.18 & 86.02 & \multirow{3}{*}{$<0.001$} \\
\hline (ii) One & 661 & 89.6 & 87.25 & 91.95 & 69.4 & 65.28 & 73.52 & \\
\hline (iii) $\geq 2$ & 598 & 75.8 & 72.27 & 79.33 & 47.5 & 43.19 & 51.81 & \\
\hline \multicolumn{9}{|l|}{ Urological complications } \\
\hline (i) No & 1816 & 87.0 & 85.43 & 88.57 & 65.9 & 63.35 & 68.45 & \multirow{2}{*}{0.449} \\
\hline (ii) Yes & 151 & 83.6 & 77.52 & 89.68 & 61.4 & 51.99 & 70.81 & \\
\hline \multicolumn{9}{|l|}{ Post-transplant hypertension } \\
\hline (i) Normotensive & 758 & 86.2 & 83.46 & 88.94 & 75.7 & 71.58 & 79.82 & \multirow{3}{*}{$<0.001$} \\
\hline (ii) Sustained hypertension & 809 & 88.0 & 85.65 & 90.45 & 65.9 & 62.18 & 69.62 & \\
\hline (iii) Newly developed hypertension & 400 & 85.7 & 82.17 & 89.23 & 56.0 & 50.71 & 61.29 & \\
\hline
\end{tabular}

explanation. However, patient and graft outcomes from older living kidney donors were reported as similar to those from younger donors despite lower GFR [15].

In an earlier report, it was observed that when the combined number of HLA-A, -B and -DR mismatches was considered, the influence of the degree of HLA incompatibility was significant [16]. In the current study, such an impact was not seen which is in agreement with a recently published report [9]. The increased number of patients who received a calcineurin inhibitor can provide an explanation. Nevertheless, the best outcomes, by far, were obtained from HLA identical siblings. Results of graft survival of kidneys obtained from 1-haplotype and 2-haplotype mismatched pairs were not different. This is in agreement with data from the collaborative transplant study (CTS) [17].

Evidence was provided that a tacrolimus-based triple therapy provided the best probability of graft survival at ten years. A similar finding was also reported by the CTS [16]. In their study, a combination of tacrolimus/azathioprine (Tac/Aza) provided a better result than that of tacrolimus/ mycophenolate mofetil (Tac/MMF). A possible explanation is that while Tac/MMF can provide a more potent immunosuppression, Tac/Aza is associated with a reduced risk of infectious complications [18].

Our data demonstrate that the incidence and number of acute rejection episodes encountered during the first 3 months after transplantation had a significant negative impact on graft survival. This is also reflected in the inverse relationship between graft survival and the total dose of corticosteroids during the same time period. A long list of predisposing factors for acute rejection can be compiled. Nevertheless, there are no reliable method(s) to predict whether a given patient will have a rejection. On this basis, it was suggested that the routine utilization of an induction regimen could be of benefit. To this end, the use of an interleukin2 receptor antibodies is attractive because of their potential role in the prevention of rejection with almost negligible side effects $[19,20]$. In a prospective randomized controlled study with an extended follow-up, Sheashaa and associates reported that basiliximab induction reduces the incidence of acute rejection significantly. However, a noticeable effect on the long-term renal transplant outcome was not seen [21]. 
TABLE 4: Cox proportional hazard analysis.

\begin{tabular}{|c|c|c|c|c|}
\hline Characteristic & $\begin{array}{c}\text { Regression estimate } \\
(B)\end{array}$ & S.E. & $\begin{array}{c}\text { Relative risk } \\
\operatorname{Exp}(B)(95 \% \mathrm{CI})\end{array}$ & $P$ value \\
\hline \multicolumn{5}{|l|}{ Donor's age (years) } \\
\hline$<30$ & - & - & 1 & \\
\hline $31-40$ & -0.049 & 0.097 & $0.952(0.788,1.150)$ & 0.609 \\
\hline $41-50$ & 0.326 & 0.109 & $1.385(1.120,1.714)$ & 0.003 \\
\hline$>50$ & 0.487 & 0.145 & $1.628(1.224,2.164)$ & $<0.001$ \\
\hline \multicolumn{5}{|l|}{ Genetic considerations } \\
\hline (i) HLA-ID siblings & - & - & 1 & \\
\hline (ii) 1-haplotype MM (R) & 0.059 & 0.203 & $1.099(0.739,1.635)$ & 0.641 \\
\hline (iii) 2-haplotype MM (R + UR) & 0.404 & 0.211 & $1.498(0.990,2.267)$ & 0.056 \\
\hline \multicolumn{5}{|c|}{ No. of acute rejection episodes (during first 3 months) } \\
\hline (i) No & - & - & 1 & \\
\hline (ii) One & 0.779 & 0.130 & $2.218(1.689,2.814)$ & $<0.001$ \\
\hline (iii) $\geq 2$ & 1.559 & 0.131 & $4.754(3.737,6.141)$ & $<0.001$ \\
\hline \multicolumn{5}{|l|}{ Total steroid dose (during first 3 months) } \\
\hline$<5 \mathrm{gm}$ & - & - & 1 & \\
\hline $5-10 \mathrm{gm}$ & -0.363 & 0.100 & $0.696(0.572,0.846)$ & 0.001 \\
\hline$>10 \mathrm{gm}$ & -0.294 & 0.135 & $0.745(0.572,0.972)$ & 0.030 \\
\hline \multicolumn{5}{|l|}{ Primary immunosuppression } \\
\hline (i) Aza-based (dual therapy) & - & - & 1 & \\
\hline (ii) CsA-based (dual therapy) & -0.022 & 0.139 & $0.978(0.744,1.285)$ & 0.872 \\
\hline (iii) CsA-based (triple therapy) & -0.234 & 0.114 & $0.791(0.633,0.989)$ & 0.039 \\
\hline (iv) Tacrolimus-based (triple therapy) & -0.686 & 0.228 & $0.504(0.322,0.787)$ & 0.003 \\
\hline
\end{tabular}

The independent and significant negative influence of the cumulative dose of corticosteroids received during the first 3 months after transplantation is not a surprise. Their multiple adverse effects on the patient's blood pressure, lipid profile, and glucose tolerance are well documented $[22,23]$. Hence, studies were carried out to explore the feasibility of corticosteroid withdrawal or minimization. These had provided evidence of the clinical benefits from early or late steroid withdrawals without an untoward effect on graft survival [2426]. In a meta-analysis by Knight and Morris, they reported that steroid avoidance after renal transplantation increases the risk of acute rejection but decreases the cardiovascular risks. They concluded that such protocols are justified with current immunosuppressive protocols in low-risk recipients [27]. A similar conclusion was reported by our group when a steroid-avoidance immunosuppression regimen was utilized for low-risk live-donor transplant recipients [3].

Despite the significant improvements in graft survival achieved by the introduction of calcineurin inhibitors, the nephrotoxicity of these agents had raised concern [28, 29]. In a longitudinal histological study, it was demonstrated that long-term calcineurin inhibitor nephrotoxicity is common and characterized by increasing small vessel-narrowing and progressive ischemic glomerulosclerosis [30]. This nephrotoxicity can account for the fact that reduction of early episodes of acute rejection did not result in a corresponding improvement in the long-term outcome. In our study, the excellent results obtained at 5 years after transplantation, followed by a steady decline in graft survival. This can be, at least partially, due to calcineurin inhibitor-induced nephrotoxicity. Several studies explored the possibility of calcineurininhibitor sparing and/or steroid avoidance immunosuppressive regimens. The best maintenance immunosuppressive regimen with calcineurin inhibitor and/or steroid sparing is a work in progress and awaits a longer follow-up. One strategy employs the utilization of a powerful agent for induction to be followed by withdrawal of the calcineurin inhibitor after a predetermined time. We have conducted a pilot study to determine the feasibility of steroid and calcineurin inhibitorfree regimen following live-donor kidney transplantation [31]. Alemtuzumab was used for induction. Tacrolimus was given for the first 2 months and replaced by sirolimus thereafter. Out of 41 recipients, 31 are enjoying steroid-free regimen of whom 21 patients are calcineurin inhibtor-free as well. This regimen proved to be effective with good patient acceptance and excellent patient and graft survival. The reported followup period of $28.3 \pm 2.1$ months is short. An extended observation period is necessary before making a final judgment.

In a previous report [32], the incidence of malignancy among our recipients was 3.9\%. Kaposi sarcoma was the common type, and posttransplant lymphoproliferative disorder (PTLD) was the second most common one. 


\section{Conclusions}

Factors affecting graft survival following renal transplantation from living donors were studied by univariate as well as multivariate analysis. Five variables were identified as factors with an independent impact on graft survival. Excellent outcomes were obtained at 5 years following transplantation. Thereafter, a steady decline was observed presumably as a result of chronic allograft nephropathy. Since the current immunosuppressive regimens play a central role in its pathogenesis, relentless efforts should be made to define the optimal steroid and/or calcineurin inhibitors sparing protocols.

\section{References}

[1] M. A. Ghoneim, M. A. Sobh, A. A. Shokeir et al., "Prospective randomized study of azathioprine versus cyclosporin in livedonor kidney transplantation," American Journal of Nephrology, vol. 13, no. 6, pp. 437-441, 1993.

[2] M. A. Ghoneim, M. A. Sobh, A. A. Shokeir, M. A. Bakr, A. ElSherif, and M. A. Foda, "Prospective randomized study of triple Vs conventional immunosuppression in living-donor kidney transplantation," Transplantation Proceedings, vol. 25, no. 3, pp. 2243-2245, 1993.

[3] A. H. Nematalla, M. A. Bakr, O. A. Gheith et al., "Steroidavoidance immunosuppression regimen in live-donor renal allotransplant recipients: a prospective, randomized controlled study," Experimental and Clinical Transplantation, vol. 5, no. 2, pp. 673-679, 2007.

[4] A. F. Hamdy, M. A. Bakr, and M. A. Ghoneim, "Long-term efficacy and safety of a calcineurin inhibitor-free regimen in live-donor renal transplant recipients," Journal of the American Society of Nephrology, vol. 19, no. 6, pp. 1225-1232, 2008.

[5] J. E. Tuttle-Newhall, S. M. Krishnan, M. F. Levy et al., "Organ donation and utilization in the United States: 1998-2007," American Journal of Transplantation, vol. 9, no. 4, part 2, pp. 879-893, 2009.

[6] DSO report on organ donation and transplantation in Germany 2000, pp. 45-46.

[7] S. Lee, J. Kim, M. Shin et al., "Comparison of outcomes of living and deceased donor kidney grafts surviving longer than 5 years in Korea," Transplantation Proceedings, vol. 42, no. 3, pp. 775777, 2010 .

[8] J. I. Roodnat, I. C. van Riemsdijk, P. G. H. Mulder et al., "The superior results of living-donor renal transplantation are not completely caused by selection or short cold ischemia time: a single-center, multivariate analysis," Transplantation, vol. 75, no. 12, pp. 2014-2018, 2003.

[9] S. V. Fuggle, J. E. Allen, R. J. Johnson et al., "Factors affecting graft and patient survival after live donor kidney transplantation in the UK," Transplantation, vol. 89, no. 6, pp. 694-701, 2010.

[10] J. A. Lowell, D. C. Brennan, S. Shenoy et al., "Living-unrelated renal transplantation provides comparable results to livingrelated renal transplantation: a 12-year single-center experience," Surgery, vol. 119, no. 5, pp. 538-543, 1996.

[11] Y. Futagawa, K. Waki, D. W. Gjertson, and P. I. Terasaki, "Livingunrelated donors yield higher graft survival rates than parental donors," Transplantation, vol. 79, no. 9, pp. 1169-1174, 2005.

[12] M. El-Mekresh, Y. Osman, B. Ali-El-Dein, T. El-Diasty, and M. A. Ghoneim, "Urological complications after living-donor renal transplantation," BJU International, vol. 87, no. 4, pp. 295-306, 2001.

[13] N. Issa, B. Stephany, R. Fatica et al., "Donor factors influencing graft outcomes in live donor kidney transplantation," Transplantation, vol. 83, no. 5, pp. 593-599, 2007.

[14] K. Noppakun, F. G. Cosio, P. G. Dean, S. J. Taler, R. Wauters, and J. P. Grande, "Living donor age and kidney transplant outcomes," American Journal of Transplantation, vol. 11, no. 6, pp. 1279-1286, 2011.

[15] L. S. Peña De La Vega, A. Torres, H. E. Bohorquez et al., "Patient and graft outcomes from older living kidney donors are similar to those from younger donors despite lower GFR," Kidney International, vol. 66, no. 4, pp. 1654-1661, 2004.

[16] M. A. Ghoneim, M. A. Bakr, N. Hassan et al., "Live-donor renal transplantation at the Urology \& Nephrology Center of Mansoura: 1976-1998," Clinical Transplants, pp. 167-178, 2001.

[17] Collaborative Transplant Study, University of Heidelberg, Germany, January 2012, http://www.ctstransplant.org.

[18] R. Weimer, S. Deisz, H. Dietrich et al., "Impact of maintenance immunosuppressive regimens-balance between graft protective suppression of immune functions and a near physiological immune response," Transplant International, vol. 24, no. 6, pp. 596-609, 2011.

[19] B. Nashan, R. Moore, P. Amlot, A. G. Schmidt, K. Abeywickrama, and J. P. Soulillou, "Randomised trial of basiliximab versus placebo for control of acute cellular rejection in renal allograft recipients," The Lancet, vol. 350, no. 9086, pp. $1193-$ 1198, 1997.

[20] P. A. Keown, R. Balshaw, S. Khorasheh et al., "Meta-analysis of basiliximab for immunoprophylaxis in renal transplantation," BioDrugs, vol. 17, no. 4, pp. 271-279, 2003.

[21] H. A. Sheashaa, M. A. Bakr, R. H. Rashad, A. M. Ismail, M. A. Sobh, and M. A. Ghoneim, "Ten-year follow-up of basiliximab induction therapy for live-donor kidney transplant: a prospective randomized controlled study," Experimental and Clinical Transplantation, vol. 9, no. 4, pp. 247-251, 2011.

[22] I. Lemieux, I. Houde, A. Pascot et al., "Effects of prednisone withdrawal on the new metabolic triad in cyclosporine-treated kidney transplant patients," Kidney International, vol. 62, no. 5, pp. 1839-1847, 2002.

[23] G. Opelz, B. Döhler, G. Laux, and Collaborative Transplant Study, "Long-term prospective study of steroid withdrawal in kidney and heart transplant recipients," American Journal of Transplantation, vol. 5, no. 4 I, pp. 720-728, 2005.

[24] E. S. Woodle, M. R. First, J. Pirsch et al., "A prospective, randomized, double-blind, placebo-controlled multicenter trial comparing early (7 day) corticosteroid cessation versus longterm, low-dose corticosteroid therapy," Annals of Surgery, vol. 248, no. 4, pp. 564-577, 2008.

[25] A. Lightner, W. Concepcion, and P. Grimm, "Steroid avoidance in renal transplantation," Current Opinion in Organ Transplantation, vol. 16, no. 5, pp. 477-482, 2011.

[26] J. Pascual, "Steroid avoidance or withdrawal in kidney transplantation," Current Opinion in Organ Transplantation, vol. 16, no. 6, pp. 600-605, 2011.

[27] S. R. Knight and P. J. Morris, "Steroid avoidance or withdrawal after renal transplantation increases the risk of acute rejection but decreases cardiovascular risk. A meta-analysis," Transplantation, vol. 89, no. 1, pp. 1-14, 2010.

[28] G. Remuzzi and N. Perico, "Cyclosporine-induced renal dysfunction in experimental animals and humans," Kidney International, vol. 52, pp. S70-S74, 1995. 
[29] B. D. Myers and L. Newton, "Cyclosporine-induced chronic nephropathy: an obliterative microvascular renal injury," Journal of the American Society of Nephrology, vol. 2, supplement 1, pp. S45-S52, 1991.

[30] B. J. Nankivell, R. J. Borrows, C. L. Fung, P. J. O’Connell, R. D. M. Allen, and J. R. Chapman, "The natural history of chronic allograft nephropathy," The New England Journal of Medicine, vol. 349, no. 24, pp. 2326-2333, 2003.

[31] A. F. Refaie, K. M. Mahmoud, A. M. Ismail, H. A. Sheashaa, A. I. Kamal, and M. A. Ghoneim, "Alemtuzumab preconditioning allows steroid-calcineurin inhibitor-free regimen in live-donor kidney transplant," Experimental and Clinical Transplantation, vol. 9, no. 5, pp. 295-301, 2011.

[32] A. F. Donia, A. Mostafa, H. Refaie, M. El-Baz, M. M. Kamal, and M. A. Ghoneim, "Postkidney transplant malignancy in Egypt has a unique pattern: a three-decade experience," Transplantation, vol. 86, no. 8, pp. 1139-1142, 2008. 


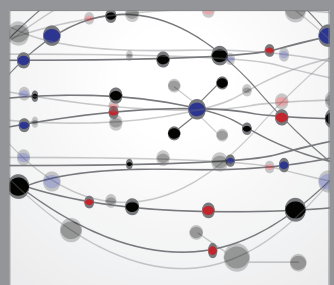

The Scientific World Journal
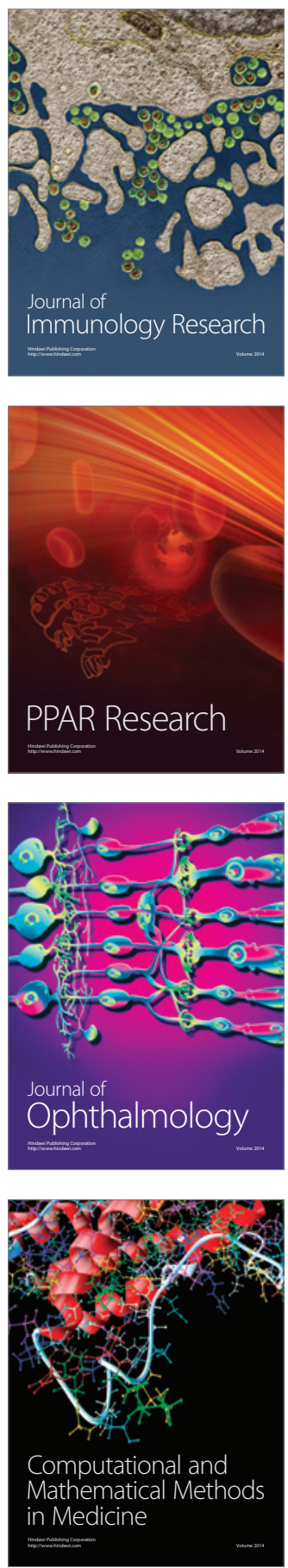

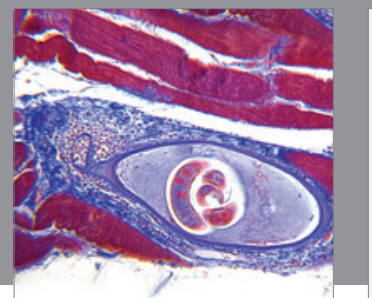

Gastroenterology

Research and Practice
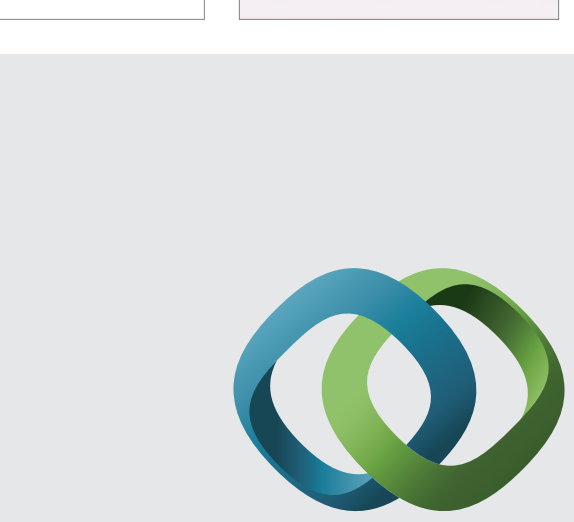

\section{Hindawi}

Submit your manuscripts at

http://www.hindawi.com
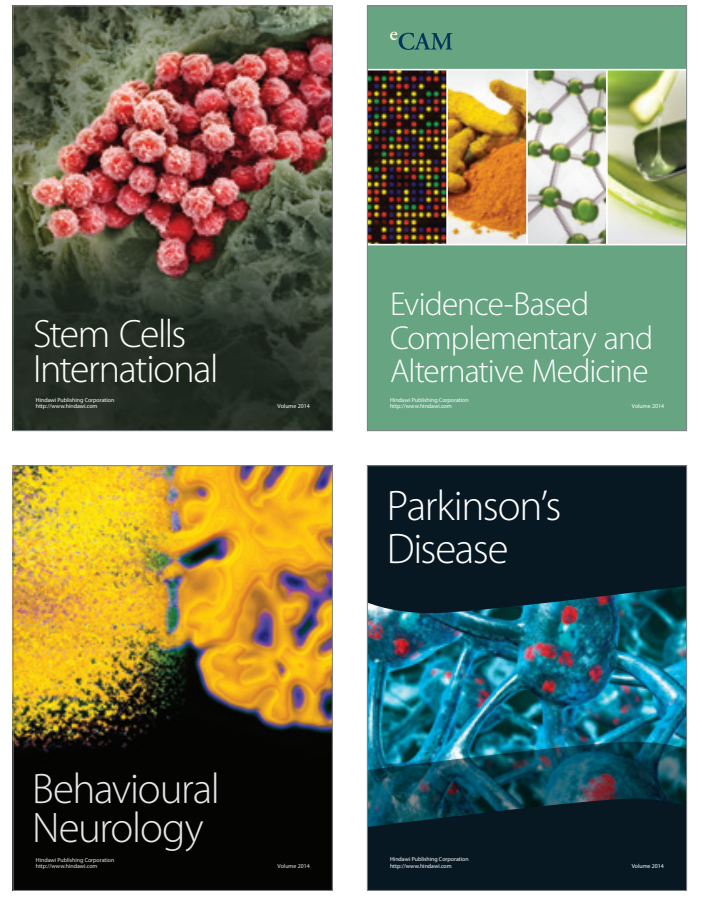
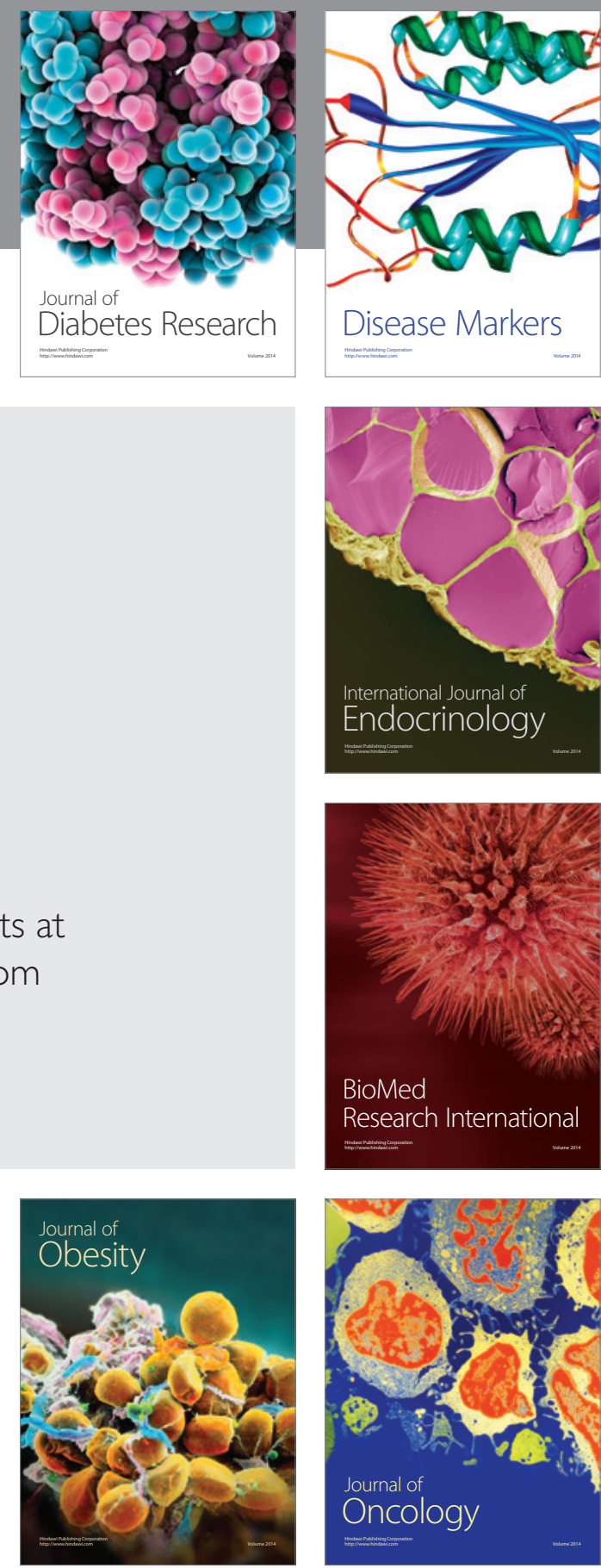

Disease Markers
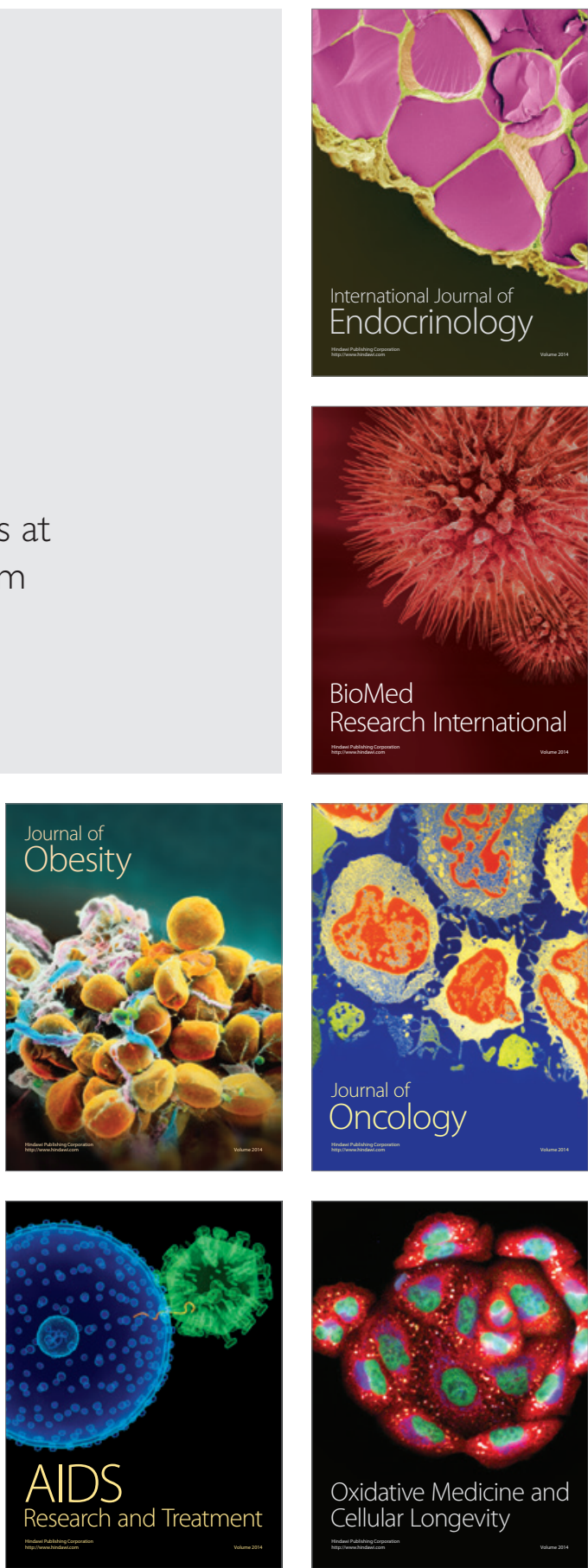